\title{
Renal cell carcinoma with venous extension: prediction of inferior vena cava wall invasion by MRI
}

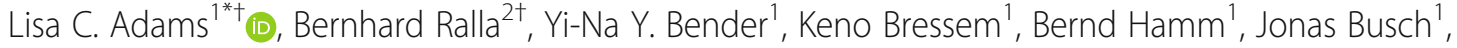 \\ Florian Fuller ${ }^{3+}$ and Marcus R. Makowski ${ }^{1+}$
}

\begin{abstract}
Background: Renal cell carcinoma (RCC) are accompanied by inferior vena cava (IVC) thrombus in up to 10\% of the cases, with surgical resection remaining the only curative option. In case of IVC wall invasion, the operative procedure is more challenging and may even require IVC resection. This study aims to determine the diagnostic performance of contrast-enhanced magnetic resonance imaging (MRI) for the assessment of wall invasion by IVC thrombus in patients with RCC, validated with intraoperative findings.

Methods: Data were collected on 81 patients with RCC and IVC thrombus, who received a radical nephrectomy and vena cava thrombectomy between February 2008 and November 2017. Forty eight patients met the inclusion criteria. Sensitivity and specificity as well as the positive and negative predictive values were calculated for preoperative MRI, based on the assessments of the two readers for visual wall invasion. Furthermore, a logistic regression model was used to determine if there was an association between intraoperative wall adherence and IVC diameter.

Results: Complete occlusion of the IVC lumen or vessel breach could reliably assess IVC wall invasion with a sensitivity of 92.3\% (95\%-Cl: 0.75-0.99) and a specificity of 86.4\% (95\%-Cl: 0.65-0.97) (Fisher-test: $p$-value< 0.001). The positive predictive value (PPV) was 88.9\% (95\%-Cl: 0.71-0.98) and the negative predictive value reached 90.5\% (95\%-Cl: 0.70-0.99). There was an excellent interobserver agreement for determining IVC wall invasion with a kappa coefficient of 0.90 (95\%Cl: 0.79-1.00).

Conclusions: The present study indicates that standard preoperative MR imaging can be used to reliably assess IVC wall invasion, evaluating morphologic features such as the complete occlusion of the IVC lumen or vessel breach. Increases in IVC diameter are associated with a higher probability of IVC wall invasion.
\end{abstract}

Keywords: Renal cell carcinoma, Inferior vena cava thrombus, Magnetic resonance imaging, Preoperative planning, Sensitivity and specificity

\section{Background}

Renal cell carcinoma (RCC) represent approximately 2$3 \%$ of all tumors and show a propensity for vascular growth with up to $10 \%$ of patients developing an inferior vena cava (IVC) thrombus [1, 2]. IVC wall invasion is a negative prognostic factor [3], whereby positive renal or caval vein margins are associated with worse survival outcomes $[4,5]$. To date, surgical resection remains the only curative option, offering a 5-year-survival of up to $40-65 \%$

\footnotetext{
* Correspondence: Lisa.adams@charite.de

${ }^{\dagger}$ Equal contributors

'Department of Radiology, Charité, Charitéplatz 1, 10117 Berlin, Germany

Full list of author information is available at the end of the article
}

for RCC with intravascular growth, which is reduced in cases with IVC wall invasion [6-8].

In case of IVC wall invasion, surgery is more challenging, because it may necessitate segmental resection or even prosthetic replacement to prevent postoperative recurrence or venous insufficiency $[9,10]$. The need for segmental resection or prosthetic replacement is typically determined intraoperatively. Therefore, the ability to predict IVC invasion preoperatively would be a clear advantage in terms of preoperative planning and a priori patient information.

High quality diagnostic imaging is a cornerstone of preoperative planning and management. With regard to the presence and extent of IVC invasion, MR is a powerful 
and accurate tool and is suggested to be more reliable than computed tomography (CT) [11]. However, data on the prediction of venous wall invasion by preoperative imaging are sparse. There have been only a limited number of studies - most of them with older generation MR scanners and a small number of patients - investigating the ability of CT or MRI to assess the extent of wall invasion and vena caval tumor extension [7, 9, 12-14]. While breach of the vessel wall with tumor signal on both sides of the vessel wall has been demonstrated to be a reliable sign of IVC wall invasion $[9,15]$, contact of the IVC thrombus with the vessel wall could not be established as a reliable predictor so far.

\section{Methods}

In the present study, we aimed to evaluate the accuracy of preoperative standard MRI for determining or ruling out wall invasion of the IVC, based on morphologic features such as vessel wall contact or vessel wall breach, with imaging findings being validated with intraoperative results. Furthermore, we sought to test for the potential association between wall invasion and IVC diameter or thrombus enhancement.

\section{Study design and population}

This retrospective study was approved by the Institutional Review Board. Between February 2008 and November 2017, 81 patients with histologically proven RCC and IVC thrombus received a radical nephrectomy and vena cava thrombectomy with an intraoperative assessment of IVC wall invasion. Of these patients, 48 patients obtained a preoperative MRI examination with a clinical routine protocol at a $1.5 \mathrm{~T}$ unit and could be included in our analysis, aiming to validate in vivo findings of IVC wall invasion with intraoperative findings.

The patient sample consisted of a total of 48 patients (10 women and 38 men, aged 38-79, mean 64.9 \pm 9.8 ). The median time between the preoperative imaging and the date of surgery was 16.1 ( \pm 13.3$)$ days. With regard to the composition of the thrombus, there were $8 \mathrm{pa}-$ tients with bland thrombus (0 cases IVC wall invasion), 19 patients with tumor thrombus (16 cases with wall invasion) and 21 patients with mixed content (10 cases with wall invasion), whereby mixed content refers to a coexistence of bland thrombus and tumor thrombus. Circumferential cavectomy with prosthetic replacement of the IVC was performed in only 3 of the 48 patients (6.3\%), whereas the other patients received a reconfiguration with continuous suturing. In the 3 cases with circumferential cavectomy, IVC tumor invasion was histologically confirmed. In 9 of the 48 patients (18.8\%) with level IV thrombi, a cardiopulmonary bypass had to be used. During histological examination, 40 of the patients were revealed to have a clear cell RCC, seven patients showed papillary carcinomas and one patients had an undifferentiated renal carcinoma, which could not be clearly classified. An overview of the patients' characteristics is provided by Table 1 .

\section{Imaging protocol}

The MRI examinations from our hospital were performed on $1.5 \mathrm{~T}$ units (Aera/Avanto/Symphony/Sonata, Siemens Medical Solutions, Erlangen, Germany) with dedicated body-phased-array coils. All patients underwent a clinical routine imaging of the kidneys at $1.5 \mathrm{~T}$, which included transverse, coronal and sagittal T2 half Fourier single-shot turbo spin echo sequences (HASTE), unenhanced axial 3D gradient echo pulse T1-weighted (FLASH) images, a T1- FLASH angiography, obtained prior to and after the intravenous administration of contrast agents, and a fat saturated volumetric interpolated breath-hold examination (VIBE) T1 3D sequence (see Table 2 for tabulated magnetic resonance imaging parameters).

\section{Level of IVC extent, tumor thrombus enhancement and IVC diameter}

Level of IVC extent was stratified following the classification of tumor thrombus level according to the Mayo

Table 1 Characteristics of the Study Population

\begin{tabular}{ll}
\hline Number of patients & 48 \\
Number of men/women & $38 / 10$ \\
Mean age at surgery (range; SD) & $64.9(38-79 ; 9.8)$ \\
Involvement of the right kidney (number) & 37 \\
Thrombus level (number, \%) & \\
I & $9(18.8)$ \\
II & $17(35.4)$ \\
III & $13(27.1)$ \\
IV & $9(18.8)$ \\
Fuhrman grade (number, \%) & \\
1 & $2(4.3)$ \\
2 & $19(40.4)$ \\
3 & $18(38.3)$ \\
4 & $8(17.0)$ \\
TNM classification (number, \%) & \\
T1 & $1(2.1)$ \\
T2 & $1(2.1)$ \\
T3a & $15(31.2)$ \\
T3b & $22(45.8)$ \\
T3c & $7(14.6)$ \\
Number of clear cell carcinoma (\%) & $2(4.2)$ \\
Pumber of papillary carcinoma (\%) & $40(83.3)$ \\
\hline
\end{tabular}


Table 2 Tabulated imaging parameters of the magnetic resonance sequences

\begin{tabular}{|c|c|c|c|c|c|c|}
\hline Type of acquisition & T2 HASTE axial & T2 HASTE ${ }^{a}$ coronary & T2 TSE $^{b}$ axial $(P A C E)^{b}$ & T1 FLASH ${ }^{c}$ & Angiography T1 FLASH & $\mathrm{T} 1 \mathrm{VIBE}^{\mathrm{d}}$ \\
\hline Repetition time, TR (ms) & 800 & 800 & 2430 & 186 & 2.88 & 4.74 \\
\hline Echo time, TE (ms) & 94 & 89 & 79 & 4.76 & 0.98 & 2.38 \\
\hline Field of view (FOV) & $340 \times 340$ & $400 \times 400$ & $340 \times 340$ & $340 \times 340$ & $500 \times 500$ & $373 \times 373$ \\
\hline Matrix size & $320 \times 320$ & $320 \times 320$ & $320 \times 320$ & $320 \times 320$ & $512 \times 512$ & $320 \times 320$ \\
\hline Slice thickness (mm) & 5 & 5 & 4 & 4 & 1.4 & 3 \\
\hline Pixel bandwidth (Hz/pixel) & 300 & 422 & 260 & 260 & 440 & 400 \\
\hline Acquisition mode & $2 \mathrm{D}$ & $2 \mathrm{D}$ & $2 \mathrm{D}$ & $2 \mathrm{D}$ & $3 \mathrm{D}$ & $3 \mathrm{D}$ \\
\hline Flip angle $\left(^{\circ}\right)$ & 180 & 170 & 180 & 70 & 25 & 10 \\
\hline Voxel size & $1.3 \times 1.1 \times 4.0$ & $1.7 \times 1.3 \times 5.0$ & $1.5 \times 1.1 \times 4.0$ & $1.4 \times 1.1 \times 4.0$ & $1.6 \times 1.0 \times 1.4$ & $1.7 \times 1.2 \times 3.0$ \\
\hline
\end{tabular}

aHalf Fourier Single-shot Turbo-spin Echo sequence

${ }^{\mathrm{b}}$ Turbo Spin Echo with Prospective Acquisition Correction

${ }^{C}$ Fast low-angle shot magnetic resonance imaging

${ }^{d}$ Volumetric Interpolated Breath-hold Examination

staging system [16]: Level I refers to thrombi that extend into the IVC to no more than $2 \mathrm{~cm}$ above the renal vein. Level II represents IVC thrombi extending into into the IVC to more than $2 \mathrm{~cm}$ above the renal vein but not to the hepatic vein, whereby Levels I and II make up for approximately $50 \%$ of the thrombi. Level III IVC thrombi are defined as extending above the hepatic veins, but below the diaphragm, making up for about $40 \%$. Level IV IVC thrombi extend above the diaphragm or into the right atrium and represent approximately $10 \%$ [17]. IVC thrombus levels for all patients were assessed at two time points, first, during preoperative imaging, and second by exploration during surgery.

With regard to the composition of IVC thrombus, an image-based differentiation was performed for bland thrombus, which was diagnosed, when there was no thrombus enhancement, tumor thrombus, which was assumed when the signal intensity was similar to that of the RCC and mixed content, which included both features and could e.g. also refer to a tumor thrombus covered by clot.

The maximum IVC diameters were measured on an axial section in two directions, which were perpendicular to each other.

\section{Image analysis}

All of the images were analyzed by use of PACS workstations (Centricity Radiology; GE Healthcare). All MRI images were evaluated by two radiologists blinded to the surgical and pathological findings and to the observations of the other in randomized order and in two different reading sessions, which were separated by a period of 2 weeks. IVC thrombi were analyzed based on the following properties: upper extent (infrahepatic, intrahepatic, infra-diaphragmatic or supradiaphragmatic), thrombus enhancement, IVC diameter and wall invasion. If the IVC thrombus could clearly be delineated from the vessel wall and if there was no thickening or altered signal of the low-intensity vessel wall, invasion was assumed to be absent. If there was a contact of the IVC thrombus with or even a visual breach of the IVC wall, IVC wall invasion was assumed to be present. More specifically, contact to the vessel wall referred to a loss of delineation between thrombus and vessel wall with complete occlusion of the vessel and blood signal loss in the affected area. Consequently, a diagnosis of wall invasion was made, when the tumor showed contact with the vessel wall or, if there was a breach or extension through the vessel wall [9]. Agreement between the two observers was also assessed. Finally, imaging findings were validated with intraoperative findings.

\section{Intraoperative evaluation and procedure}

Intraoperatively, wall invasion was reported, if the IVC thrombus showed any adherence to the IVC wall. Absence of IVC wall invasion was confirmed, if the caval thrombus could be easily removed.

If the IVC does not show any signs of advanced invasion intraoperatively and there is no evidence of the resection compromising the IVC lumen, the standard operative procedure at our institution involves a combination of thrombectomy with subsequent cavorrhaphy, using continuous polypropylene suturing. If there is advanced tumorous invasion with a breach of the vessel wall IVC resection, either segmental or circumferential, become necessary.

\section{Statistical analysis}

Statistical analysis was performed with "R" Statistical Software (Version 3.2.2, R Development Core Team, 2015). Variables were expressed as means \pm standard deviations. Sensitivity and specificity as well as the positive and negative predictive values were calculated based on the assessments of the two readers for visual wall invasion. In case of a differing assessment, the opposite of the reference standard (intraoperative finding) was assumed in order to avoid an overestimation of the diagnostic performance. Cohen's kappa coefficient was used to measure interobserver 
agreement for categorical variables (invasion/no invasion). The intraclass coefficient (ICC) was used to assess interobserver and intermodality reliability for continuous data. Interobserver and intermodality reliability was considered poor for ICC/kappa values less than 0.40 , fair for values from $0.40-0.59$, good for values from $0.60-0.74$ and excellent for values above 0.75 . Furthermore, a logistic regression model was used to determine if there was an association between intraoperative wall adherence and IVC diameter. Fisher's exact test was used to assess if thrombus enhancement showed a significant association with IVC wall invasion. A $p$-value $<0.05$ was considered statistically significant.

\section{Results}

All of the 48 patients underwent extended nephrectomy and thrombectomy, with the information available from surgery being used to confirm IVC wall invasion.

\section{Validation with intraoperative findings}

We found that contact of the IVC thrombus to or breach of the vessel wall could reliably diagnose wall invasion in preoperative MRI imaging with a sensitivity of 92.3\% (95\%-CI: $0.75-0.99)$ and a specificity of $86.4 \%$ (95\%-CI: 0.65-0.97) (Fisher test: p-value <0.001). The positive predictive value (PPV) was $88.9 \%$ (95\%-CI: 0 . 71-0.98) and the negative predictive value reached 90. 5\% (95\%-CI: 0.70-0.99) (refer to Table 3).

There were 26 cases of wall invasion, of which 24 were correctly identified based on contrast-enhanced MRI (see Figs. 1 and 2 for case examples). The two patients with IVC invasion, who were considered to have a non-adherent thrombus based on MRI, were revealed to have very small areas of adherence (less than $1 \mathrm{~cm}$ ) intraoperatively. In one of these cases, the assessment of the observers differed. Of the 22 cases, where intraoperative findings revealed no presence of wall invasion, 19 could be correctly identified with MRI (see Fig. 3 for case example). In the three cases, where MRI could not identify absence of wall invasion, this was mostly due to respiratory motion artifacts on the axial images, with the observers' evaluation differing in two of the cases.

Table 3 Diagnostic performance of MRI with surgery as the reference standard

\begin{tabular}{|c|c|c|c|}
\hline & Observer 1 & Observer 2 & $\begin{array}{l}\text { Observer } 1 \\
\text { and } 2 \text { combined }\end{array}$ \\
\hline Sensitivity & $0.92(0.75-0.99)$ & $0.96(0.80-1.0)$ & $0.92(0.75-0.99)$ \\
\hline Specificity & $0.95(0.77-1.0)$ & $0.86(0.65-0.97)$ & $0.86(0.65-0.97)$ \\
\hline $\begin{array}{l}\text { Negative } \\
\text { predictive value }\end{array}$ & $0.91(0.72-0.99)$ & $0.95(0.75-1.0)$ & $0.91(0.70-0.99)$ \\
\hline $\begin{array}{l}\text { Positive } \\
\text { predictive value }\end{array}$ & $0.96(0.80-1.0)$ & $0.89(0.72-0.98)$ & $0.89(0.71-0.98)$ \\
\hline
\end{tabular}

\section{Interobserver agreement for IVC wall invasion}

There was an excellent interobserver agreement for determining IVC wall invasion with a kappa coefficient of 0.95 (95\%CI: 0.85-0.98). In three cases, the two observers assessed the presence or absence of invasion differently, whereby the opposite of the reference standard (findings at surgery) was chosen in order to avoid an overestimation of the diagnostic performance (see Table 4).

Regarding IVC diameter measurements, there was also an excellent interobserver agreement with an ICC coefficient of 0.90 (95\%CI: 0.79-1.00).

\section{Association between IVC diameter and probability of IVC invasion}

Furthermore, we found that increases in IVC diameter were associated with a higher probability of IVC wall invasion, with the $\beta$-coefficient for the IVC diameter being 0.41 (standard error $+/-0.13)$. This influence also reached significance level $(p=0.015)$. Patients with IVC wall invasion showed a mean diameter of $42.96 \mathrm{~mm} \pm 8.54 \mathrm{~mm}$, while patients without wall invasion had a mean diameter $34.00 \mathrm{~mm}$ $\pm 7.25 \mathrm{~mm}$. This difference was also significant $(p=0.0 .001)$.

By contrast, intraoperative IVC wall invasion was not significantly related to the extent or level of IVC extent $(p>0.05)$.

\section{Assessment of IVC wall invasion based on MR enhancement}

A clear differentiation between invasive and noninvasive IVC thrombus based on MR enhancement patterns proved to be unfeasible, as enhancement was observed in 14 out of the 22 cases without IVC invasion, indicating a high number of false positives. However, none of the non-enhancing IVC thrombi showed signs of invasion, inferring a good estimate of the false negatives. Therefore, the association between enhancement and IVC invasion was significant (Fisher test: $p=0.001$ ). This matches the finding of a significant association between the composition of the IVC thrombus (if it was a bland thrombus/venous clot, a tumor thrombus or an association of both) and enhancement. While no enhancement could be observed in patients with bland tumor thrombus, there was enhancement in patients with tumor thrombus and mixed content.

\section{Discussion}

This study suggests that preoperative MR imaging enables a reliable determination of IVC wall invasion in patients with RCC. More specifically, complete occlusion of the IVC lumen and breach of the vessel wall are indicators of IVC invasion with a high sensitivity and specificity. Furthermore, increases in IVC diameter were associated with a higher probability of IVC wall invasion.

IVC wall invasion has recently been recognized as an independent prognostic factor for the survival of patients 


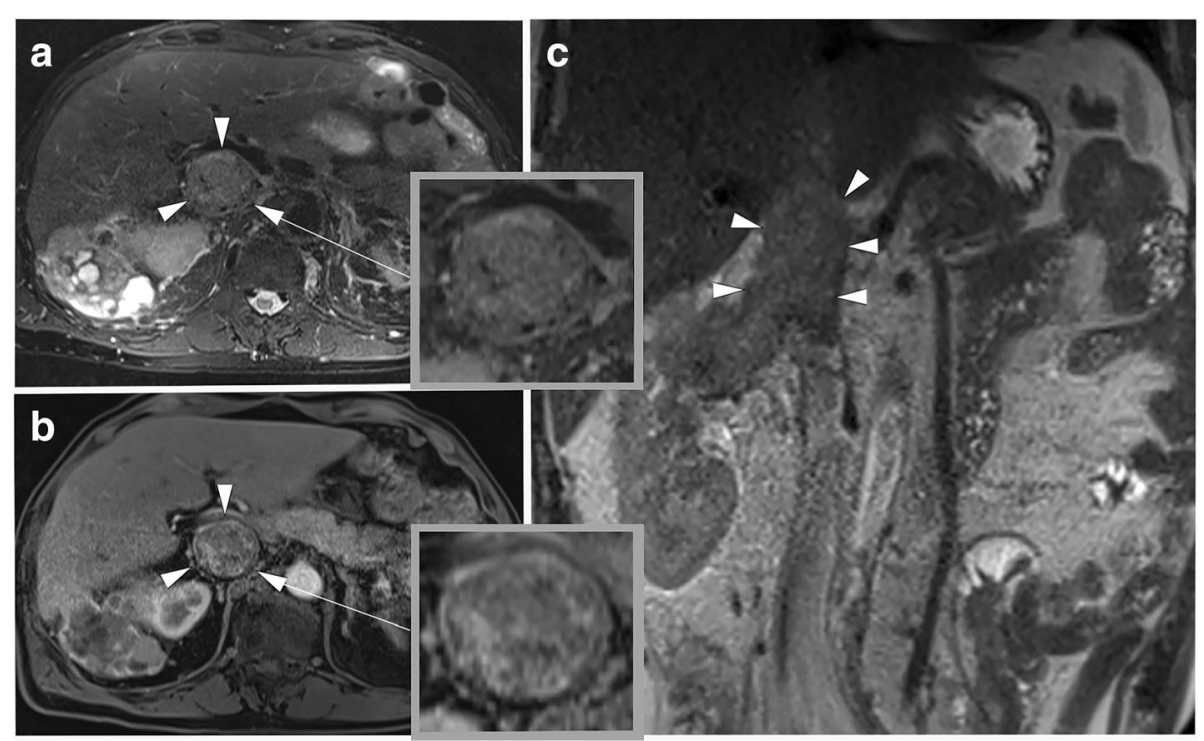

Fig. 1 Images in a 55-year old man with a clear cell renal cell carcinoma (RCC) and an inferior vena cava (IVC) tumor thrombus with wall invasion. The RCC extends from the right kidney into the suprahepatic IVC. a axial fat-saturated T2-weighted image. b T1-weighted contrastenhanced 3D GRE (VIBE) image (arterial phase) and (c), coronal T2-weighted HASTE image for anatomic reference. Note that the thrombus completely obstructs the lumen of the IVC and shows direct contact with the vessel wall $(\mathbf{a}, \mathbf{c})$. The contrast-enhanced image (b) demonstrates a heterogeneous enhancement of the tumor thrombus, and contact to, but no breach of the vessel wall, which makes IVC wall invasion likely. During extended nephrectomy, this thrombus was partly adherent the IVC and after extraction of the IVC thrombus, continuous suturing became necessary. VIBE = Volumetric interpolated breath-hold examination

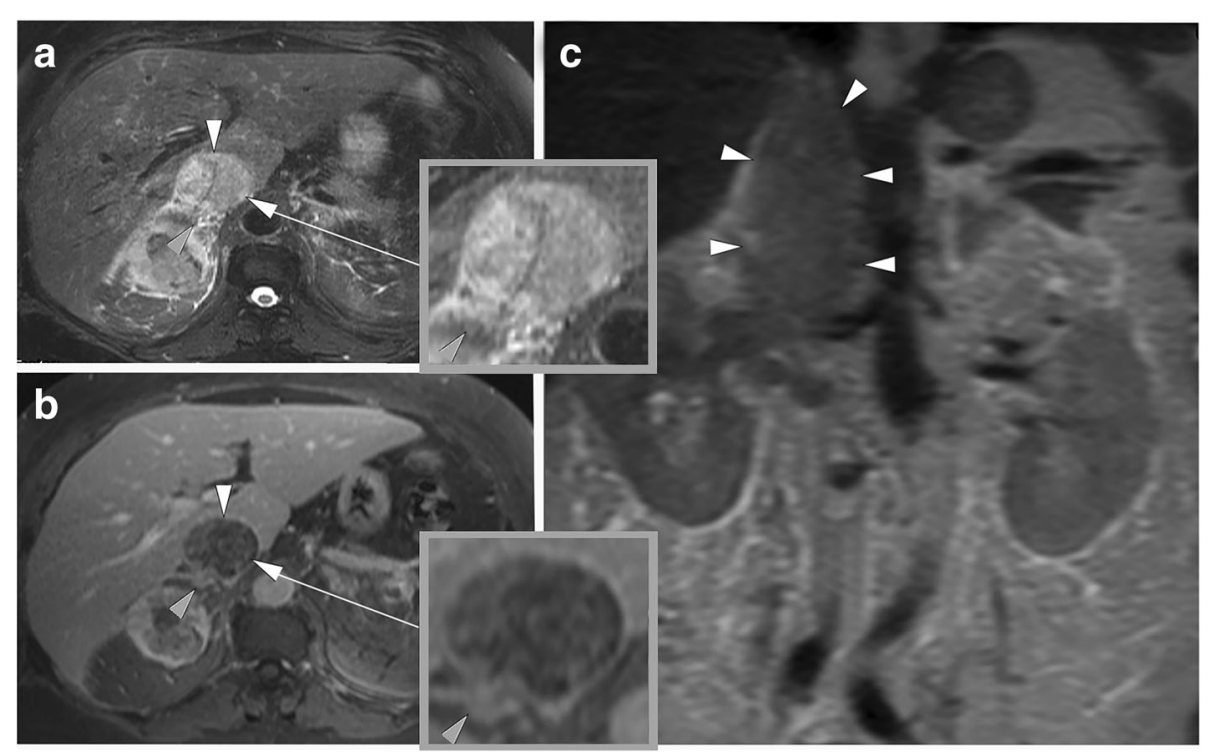

Fig. 2 Images in a 69-year old woman with a clear cell renal cell carcinoma (RCC) and an inferior vena cava (IVC) tumor thrombus with wall invasion. The RCC extends from the right kidney into the IVC and extends into the right atrium. a axial fat-saturated T2-weighted image. b T1-weighted contrast-enhanced 3D GRE (VIBE) image contrast enhanced 3D GRE image (arterial phase) and (c), coronal T2-weighted HASTE image for anatomic reference. Note that the thrombus completely obstructs the lumen of the IVC, but also seems to breach the vessel wall (a, $\mathbf{c})$. The contrast-enhanced image (b) demonstrates a heterogeneous enhancement of the tumor thrombus and a clear breach of the vessel wall (gray arrowhead), which is highly suggestive of IVC wall invasion. During extended nephrectomy, this thrombus showed strong adherence to the IVC wall and during extraction, circumferential cavectomy with vascular reconstruction became necessary. VIBE = Volumetric interpolated breath-hold examination 


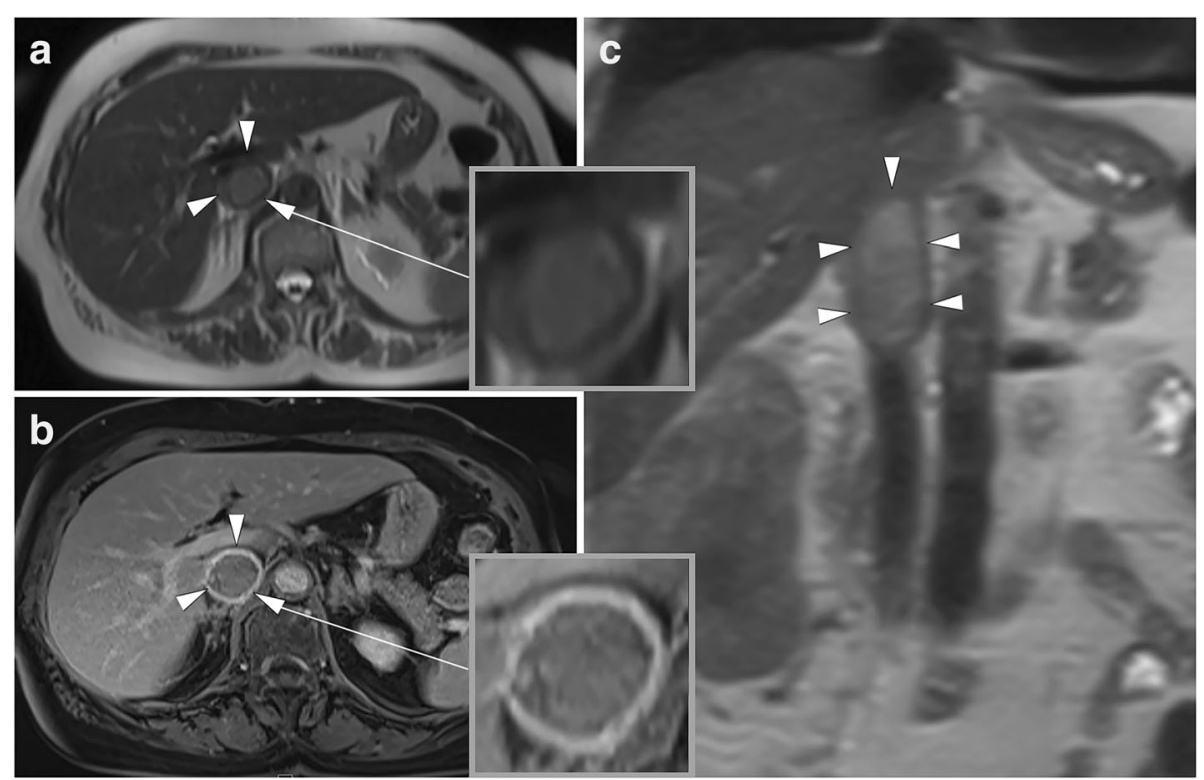

Fig. 3 Images in a 79-year old woman with a clear cell renal cell carcinoma (RCC) and bland inferior vena cava (IVC) thrombus without wall invasion. The RCC extends from the right kidney into the infrahepatic IVC. a axial T2-weighted HASTE image. b T1-weighted contrast-enhanced 3D GRE (VIBE) image and (c), the coronal T2-weighted HASTE image for anatomic reference. Note that the thrombus is floating in the IVC and that there is no complete obstruction of the caval lumen $(\mathbf{a}, \mathbf{c})$. The contrast-enhanced image (b) demonstrates that there is no enhancement of the tumor thrombus, contact to or breach of the vessel wall, so that IVC wall invasion appears unlikely. During extended nephrectomy, this thrombus could be easily removed from the IVC without necessitating segmental resection. HASTE = Half-Fourier-acquired singe-shot turbo spin echo, VIBE = Volumetric interpolated breath-hold examination

with IVC thrombi $[16,18-20]$. During surgery, IVC wall invasion requires a very challenging reconstruction beyond standard cavorrhaphy, that might include segmental resection or prosthetic replacement. However, there has been limited data with only a handful of studies and apart from the study by Psutka et al. - with small sample

Table 4 Wall invasion by inferior vena cava thrombus on MRI versus invasion determined at surgery

\begin{tabular}{lll}
\hline Observer 1 MRI & Surgery & \\
& Wall invasion & Absence of wall invasion \\
Wall invasion & 24 & 1 \\
Absence of wall invasion & 2 & 21 \\
Observer 2 MRI & Surgery & \\
& Wall invasion & Absence of wall invasion \\
Wall invasion & 25 & 3 \\
Absence of wall invasion & 1 & 19 \\
Observers 1+2 MRI & Surgery & \\
Wall invasion & Wall invasion & Absence of wall invasion \\
Absence of wall invasion & 24 & 3 \\
\hline
\end{tabular}

In three cases, the two observers assessed the presence or absence of invasion differently. In case of a differing assessment, the opposite of the reference standard (intraoperative finding) was assumed in order to avoid an overestimation of the diagnostic performance. This combined assessment (observers $1+2$ ) is shown below sizes $[9,12,14,21,22]$. With regard to MR imaging, the number of studies is even more limited, as Psutka et al., for example, chose a cross-sectional approach including both MRI and CT images, with CT having a comparably lower diagnostic performance, and using a combination of multiple radiographic parameters to predict IVC invasion [14]. By contrast, we propose a purely MR-based approach with focus on the direct detection of IVC wall invasion with clearly defined morphologic parameters.

In line with our results, previous studies found tumor signal on both sides of the vessel wall to be one of the most reliable indicators of vessel breach and of IVC wall invasion [9, 12, 21]. Furthermore, Myneni et al. proposed to use the low signal intensity line of the normal vessel wall on gradient echo (GRE) and T1-weighted images as a minor criterion for vessel wall invasion [13].

In addition, contrast enhancement of the thrombus or venous wall has been suggested as a criterion for distinguishing tumor thrombus from bland thrombus and for narrowing the diagnosis to wall invasion, the theory being that the neo-vascular bed of the tumor thrombus would adhere to the venous wall, whereas the bland thrombus would not $[9,12]$. In the present study, thrombus enhancement was not reliable for excluding caval wall invasion, as enhancement was observed in more than $60 \%$ of the cases, where no wall invasion was found intraoperatively. However, it proved to be reliable 
for excluding invasion in cases, where no IVC enhancement was observed.

Furthermore, previous studies demonstrated an association between IVC diameters and wall invasion [14, 23]. Psutka et al. considered $24 \mathrm{~mm}$ or more on the level of the renal vein ostium to be a probable indicator of advanced IVC invasion [14, 23]. In the present study, probability of IVC wall invasion also was higher with increasing IVC diameters, which appears logical, as bigger thrombi show a higher propensity for invasion.

The presence of IVC wall invasion has also been incorporated into the American Joint Committee on Cancer (AJCC) cancer staging criteria for RCC, changing a stage T3b to a stage T3c [24].

Previous research suggested a superior diagnostic accuracy of MRI in detecting the upper extent of IVC thrombus due to its intrinsic contrast superiority [25]. However, in more recent studies, the diagnostic performance of CT and MRI in staging the level of IVC thrombus has been regarded to be similar [26-29]. However, to our knowledge there has not been a systematic comparison between multidetector CT and MRI concerning the detection of IVC wall invasion yet.

Even though it has to be acknowledged, that the preoperative assessment of IVC invasion cannot replace the value of surgical exploration, morphologic MR features may be used to predict the risk for complicated inferior vena cava resection in a reproducible manner, which is also supported by the strong interobserver agreement we found. In clinical practice, surgeons can use the additional information to optimize their preoperative planning, e.g. in consultation with the vascular surgeons or scheduling of the most experienced surgeons. The derived information is especially important in patients, in whom reconstruction of the IVC beyond cavorrhaphy is probable, to determine the need for specific operative resources (e.g. cardiopulmonary bypass) in advance and also to individually improve prior patient information [15]. Intraoperatively, the a priori assessment of wall invasion can be a helpful adjunct to additional examinations such as duplex ultrasound or transesophageal echocardiography, which can be used to further characterize the mobility, consistency and the exact extension of the thrombus [14].

Recent studies have focused on diffusion weighted imaging (DWI) as an emerging technique for quantitative readouts, with the apparent diffusions coefficient (ADC) values representing tumor cellularity [30]. DWI has shown promise in preoperative cancer staging, e.g. for endometrial, cervical, bladder, rectal or gastric cancer [31-34]. Especially the introduction of reduced field-of-view (FOV) techniques has enabled an improved tumor delineation with higher spatial resolution, decreased partial volume averaging and less susceptibility distortion [35]. Future studies on the preoperative evaluation of IVC invasion could, therefore, include reduced
FOV DWI sequences to investigate whether additional information on thrombus composition may be gained. Furthermore, functional imaging, such as dynamic contrast enhanced (DCE) MRI, may improve assessment of thrombus and wall enhancement through the quantification of contrastenhancement characteristics and the evaluation of microcirculation parameters. During DCE imaging, several contrast enhancement parameters can be used to better differentiate tumor tissue. Previous research suggested, that especially the early postcontrast phase could be relevant for tumor detection [36]. By contrast, there were also studies suggesting, that there was limited additional value for diagnosis of clinically relevant cancer [37]. Two notable disadvantages of the semiquantitative parameters derived from DCE imaging is their direct estimation from the signal intensity measurements without physiological or empirical correlation and also their dependence on experimental factors such as sequence parameters or contrast dose, especially limiting their comparability and reproducibility between different sites [38].

In the present study, patients did not receive dynamic imaging, but only an early postcontrast phase. In everyday clinical practice, the time required for extensive examination protocols can be limited. Therefore, the objective of the present study was to test the performance and feasibility of a relatively short standard protocol for the preoperative evaluation of inferior vena cava wall invasion.

Regarding the results of the present study for MRI, the high sensitivity (92.3\%) indicates, that the presence of IVC invasion is rarely underestimated and the high negative predictive value $(90.5 \%)$ suggests, that if the IVC thrombus does not show any contact with the vessel wall, an IVC wall invasion can be reliably excluded. The comparably lower specificity shows, that a visual contact of the IVC thrombus with the vessel wall does not always correspond to IVC wall invasion, but still in more than $85 \%$ of the cases.

The excellent interobserver agreement for the assessment indicates the feasibility of using MR features for assessing IVC thrombus invasion in clinical practice. Complete occlusion of the IVC lumen or breach of the vessel wall may be used to predict the presence of IVC wall invasion and thus of complicated surgery.

This study has some limitations. Firstly, different MRI scanners were included over a relatively long period of time, resulting in a potential variability across scanners and imaging sessions, where the same scanner is used. Secondly, due to its retrospective design, a potential selection bias cannot be excluded. Thirdly, as intraoperative findings were used as the reference standard for wall invasion, presence of microscopic invasion cannot be excluded. Fourthly, as the present study is single center, external validation of the applied MR features is warranted. Furthermore, the addition of DWI or DCE imaging to the protocol might have further improved the preoperative assessment of IVC thrombus. Finally, MR imaging is contraindicated in some 
patients, e.g. patients with pacemakers, metallic foreign bodies or with severe claustrophobia.

\section{Conclusions}

This study indicates that standard preoperative MR imaging can be used to reliably assess IVC wall invasion, evaluating morphologic features such as the complete occlusion of the IVC lumen or vessel breach. The excellent interobserver agreement suggests an adequate reproducibility of the preoperative assessment in clinical practice. In future, MR morphologic features might be used to refine preoperative planning and improve prior patient information.

\section{Abbreviations}

CT: Computed tomography; GRE: Gradient-echo; ICC: Intraclass coefficient; IVC: Inferior vena cava; MRI: Magnetic resonance imaging; RCC: Renal cell carcinoma

\section{Acknowledgements}

LCA and BR are grateful for their participation in the $\mathrm{BIH}$ Charité - Junior Clinician Scientist Program funded by the Charité - Universitaetsmedizin Berlin and the Berlin Institute of Health. JB is participant in the $\mathrm{BIH}-$ Twinning Grant Program funded by the Charité - Universitaetsmedizin Berlin and the Berlin Institute of Health. MRM is grateful for the financial support from the Deutsche Forschungsgemeinschaft (DFG, 5943/31/41).

\section{Funding}

$\mathrm{BH}$ has received research grants for the Department of Radiology, Charité Universitätsmedizin Berlin from the following companies: 1. Abbott, 2. Actelion Pharmaceuticals, 3. Bayer Schering Pharma, 4. Bayer Vital, 5. BRACCO Group, 6. Bristol-Myers Squibb, 7. Charite research organisation $\mathrm{GmbH}, 8$. Deutsche Krebshilfe, 9. Dt. Stiftung für Herzforschung, 10. Essex Pharma, 11. EU Programmes, 12. Fibrex Medical Inc., 13. Focused Ultrasound Surgery Foundation, 14. Fraunhofer Gesellschaft, 15. Guerbet, 16. INC Research, 17. InSightec Ud., 18. IPSEN Pharma, 19. Kendlel MorphoSys AG, 20. Lilly GmbH, 21. Lundbeck GmbH, 22. MeVis Medical Solutions AG, 23. Nexus Oncology, 24. Novartis, 25. Parexel Clinical Research Organisation Service, 26. Perceptive, 27. Pfizer GmbH, 28. Philipps, 29. Sanofis-Aventis S.A, 30. Siemens, 31. Spectranetics GmbH, 32. Terumo Medical Corporation, 33. TNS Healthcare GMbH, 34 Toshiba, 35. UCB Pharma, 36. Wyeth Pharma, 37. Zukunftsfond Berlin (TSB), 38. Amgen, 39. AO Foundation, 40. BARD, 41. BBraun, 42. Boehring Ingelheimer, 43. Brainsgate, 44. PPD (Clinical Research Organisation), 45. CELLACT Pharma, 46. Celgene, 47. CeloNova BioSciences, 48. Covance, 49. DC Deviees, Ine. USA, 50. Ganymed, 51. Gilead Sciences, 52. Glaxo Smith Kline, 53. ICON (Clinical Research Organisation), 54. Jansen, 55. LUX Bioseienees, 56. MedPass, 57. Merek, 58. Mologen, 59. Nuvisan, 60. Pluristem, 61. Quintiles, 62. Roehe, 63. Sehumaeher GmbH (Sponsoring eines Workshops), 64. Seattle Geneties, 65. Symphogen, 66. TauRx Therapeuties Ud., 67. Accovion, 68. AIO: Arbeitsgemeinschaft Internistische Onkologie, 69. ASR Advanced sleep research, 70. Astellas, 71. Theradex, 72. Galena Biopharma, 73. Chiltern, 74. PRAint, 75. Inspiremd, 76. Medronic, 77. Respicardia, 78. Silena Therapeutics, 79. Spectrum Pharmaceuticals, 80. St. Jude., 81. TEVA, 82. Theorem, 83. Abbvie, 84. Aesculap, 85. Biotronik, 86. Inventivhealth, 87. ISA Therapeutics, 88 LYSARC, 89. MSD, 90. novocure, 91. Ockham oncology, 92. Premier-research 93. Psi-cro, 94. Tetec-ag, 94. Tetec-ag, 95. Winicker-norimed, 96. Achaogen Inc., 97. ADIR, 98. AstraZenaca AB, 99. Demira Inc., 100.Euroscreen S.A., 101. Galmed Research and Development Ltd., 102. GETNE, 103. Guidant Europe NV, 104. Holaira Inc., 105. Immunomedics Inc., 106. Innate Pharma, 107. Isis Pharmaceuticals Inc., 108. Kantar Health GmbH, 109. Medlmmune Inc., 110. Medpace Germany GmbH (CRO), 111. Merrimack Pharmaceuticals Inc., 112. Millenium Pharmaceuticals Inc., 113. Orion Corporation Orion Pharma, 114. Pharmacyclics Inc., 115. PIQUR Therapeutics Ltd., 116. Pulmonx International Sárl, 117. Servier (CRO), 118. SGS Life Science Services (CRO), 119. Treshold Pharmaceuticals Inc. MRM received financial support from the Deutsche Forschungsgemeinschaft (DFG, 5943/31/41/91). LCA and BR are participants in the BIH Charité - Junior Clinician Scientist Program funded by the Charité
- Universitaetsmedizin Berlin and the Berlin Institute of Health. JB is participant in the $\mathrm{BIH}$ - Twinning Grant Program funded by the Charité Universitaetsmedizin Berlin and the Berlin Institute of Health.

\section{Availability of data and materials}

The datasets used and/or analysed during the current study are available from the corresponding author on reasonable request.

\section{Authors' contributions}

MRM, LCA, FF and BR conceived of the present idea. MRM supervised the project. $L C A, Y B$ and $K B$ acquired, analyzed and interpreted the patient data regarding wall invasion by IVC thrombus (MRI versus surgical findings). LCA was a major contributor in writing the manuscript. $\mathrm{BH}, \mathrm{MRM}$, JB and FF gave technical support and conceptual advice. All authors read and revised the manuscript critically, approving the final manuscript.

\section{Ethics approval and consent to participate}

This retrospective study was approved by the Institutional Review Board (Ethikkommission der Charité, approval no. EA1/412/16), including a waiver of informed consent.

\section{Competing interests}

The authors declare that they have no competing interests.

\section{Publisher's Note}

Springer Nature remains neutral with regard to jurisdictional claims in published maps and institutional affiliations.

\section{Author details}

1Department of Radiology, Charité, Charitéplatz 1, 10117 Berlin, Germany. ²Department of Urology, Charité, Charitéplatz 1, 10117 Berlin, Germany. ${ }^{3}$ Department of Urology, Charité, Hindenburgdamm 30, 12200 Berlin, Germany.

Received: 30 January 2018 Accepted: 25 April 2018 Published online: 03 May 2018

\section{References}

1. Ljungberg B, Bensalah K, Canfield S, Dabestani S, Hofmann F, Hora M, Kuczyk MA, Lam T, Marconi L, Merseburger AS, et al. EAU guidelines on renal cell carcinoma: 2014 update. Eur Urol. 2015;67:913-24.

2. Blute ML, Leibovich BC, Lohse CM, Cheville JC, Zincke H. The Mayo Clinic experience with surgical management, complications and outcome for patients with renal cell carcinoma and venous tumour thrombus. BJU Int. 2004;94:33-41

3. Wagner B, Patard JJ, Mejean A, Bensalah K, Verhoest G, Zigeuner R, Ficarra V, Tostain J, Mulders P, Chautard D, et al. Prognostic value of renal vein and inferior vena cava involvement in renal cell carcinoma. Eur Urol. 2009:55:452-9.

4. Hatcher PA, Anderson EE, Paulson DF, Carson CC, Robertson JE. Surgical management and prognosis of renal cell carcinoma invading the vena cava. J Urol. 1991;145:20-3. discussion 23-24.

5. Rodriguez Faba O, Linares E, Tilki D, Capitanio U, Evans CP, Montorsi F Martinez-Salamanca Jl, Libertino J, Gontero P, Palou J. Impact of Microscopic Wall invasion of the renal vein or inferior vena cava on Cancer-specific survival in patients with renal cell carcinoma and tumor Thrombus: a multiinstitutional analysis from the international renal cell carcinoma-venous Thrombus consortium. Eur Urol Focus. 2017. https://doi.org/10.1016/j.euf. 2017.01.009.

6. Lambert EH, Pierorazio PM, Shabsigh A, Olsson CA, Benson MC, McKiernan JM. Prognostic risk stratification and clinical outcomes in patients undergoing surgical treatment for renal cell carcinoma with vascular tumor thrombus. Urology. 2007;69:1054-8

7. Roubidoux MA, Dunnick NR, Sostman HD, Leder RA. Renal carcinoma: detection of venous extension with gradient-echo MR imaging. Radiology. 1992;182:269-72.

8. Manassero F, Mogorovich A, Di Paola G, Valent F, Perrone V, Signori S, Boggi U, Selli C. Renal cell carcinoma with caval involvement: contemporary strategies of surgical treatment. Urol Oncol. 2011:29:745-50.

9. Aslam Sohaib SA, Teh J, Nargund VH, Lumley JS, Hendry WF, Reznek RH. Assessment of tumor invasion of the vena caval wall in renal cell carcinoma cases by magnetic resonance imaging. J Urol. 2002;167:1271-5. 
10. Zini L, Destrieux-Garnier L, Leroy X, Villers A, Haulon S, Lemaitre L, Koussa M. Renal vein ostium wall invasion of renal cell carcinoma with an inferior vena cava tumor thrombus: prediction by renal and vena caval vein diameters and prognostic significance. J Urol. 2008;179:450-4. discussion 454.

11. Kandpal H, Sharma R, Gamangatti S, Srivastava DN, Vashisht S. Imaging the inferior vena cava: a road less traveled. Radiographics. 2008;28:669-89.

12. Oto A, Herts BR, Remer EM, Novick AC. Inferior vena cava tumor thrombus in renal cell carcinoma: staging by MR imaging and impact on surgical treatment. AJR Am J Roentgenol. 1998;171:1619-24.

13. Myneni L, Hricak H, Carroll PR. Magnetic resonance imaging of renal carcinoma with extension into the vena cava: staging accuracy and recent advances. Br J Urol. 1991;68:571-8.

14. Psutka SP, Boorjian SA, Thompson RH, Schmit GD, Schmitz JJ, Bower TC, Stewart SB, Lohse CM, Cheville JC, Leibovich BC. Clinical and radiographic predictors of the need for inferior vena cava resection during nephrectomy for patients with renal cell carcinoma and caval tumour thrombus. BJU Int. 2015;116:388-96.

15. Psutka SP, Leibovich BC. Management of inferior vena cava tumor thrombus in locally advanced renal cell carcinoma. Ther Adv Urol. 2015;7:216-29.

16. Hatakeyama S, Yoneyama T, Hamano I, Murasawa H, Narita T, Oikawa M, Hagiwara K, Noro D, Tanaka T, Tanaka Y, et al. Prognostic benefit of surgical management in renal cell carcinoma patients with thrombus extending to the renal vein and inferior vena cava: 17-year experience at a single center. BMC Urol. 2013;13:47.

17. Neves RJ, Zincke H. Surgical treatment of renal cancer with vena cava extension. Br J Urol. 1987;59:390-5.

18. Chen X, Li S, Xu Z, Wang K, Fu D, Liu Q, Wang X, Wu B. Clinical and oncological outcomes in Chinese patients with renal cell carcinoma and venous tumor thrombus extension: single-center experience. World J Surg Oncol. 2015:13:14

19. Cho MC, Kim JK, Moon KC, Kim HH, Kwak C. Prognostic factor for Korean patients with renal cell carcinoma and venous tumor thrombus extension: application of the new 2009 TNM staging system. Int Braz J Urol. 2013;39:353-63.

20. Whitson JM, Reese AC, Meng MV. Population based analysis of survival in patients with renal cell carcinoma and venous tumor thrombus. Urol Oncol. 2013:31:259-63.

21. Laissy JP, Menegazzo D, Debray MP, Toublanc M, Ravery V, Dumont E, Schouman-Claeys E. Renal carcinoma: diagnosis of venous invasion with Gd-enhanced MR venography. Eur Radiol. 2000;10:1138-43.

22. Vergho DC, Loeser A, Kocot A, Spahn M, Riedmiller H. Tumor thrombus of inferior vena cava in patients with renal cell carcinoma - clinical and oncological outcome of 50 patients after surgery. BMC Res Notes. 2012;5:5.

23. Gohji K, Yamashita C, Ueno K, Shimogaki H, Kamidono S. Preoperative computerized tomography detection of extensive invasion of the inferior vena cava by renal cell carcinoma: possible indication for resection with partial cardiopulmonary bypass and patch grafting. J Urol. 1994;152:1993-6. discussion 1997

24. Edge SB, Compton CC. The American joint committee on Cancer: the 7th edition of the AJCC cancer staging manual and the future of TNM. Ann Surg Oncol. 2010;17:1471-4.

25. Hallscheidt PJ, Fink C, Haferkamp A, Bock M, Luburic A, Zuna I, Noeldge G, Kauffmann G. Preoperative staging of renal cell carcinoma with inferior vena cava thrombus using multidetector $\mathrm{CT}$ and MRI: prospective study with histopathological correlation. J Comput Assist Tomogr. 2005;29:64-8.

26. Guo HF, Song Y, Na YQ. Value of abdominal ultrasound scan, CT and MRI for diagnosing inferior vena cava tumour thrombus in renal cell carcinoma. Chin Med J. 2009;122:2299-302.

27. Gupta NP, Ansari MS, Khaitan A, Sivaramakrishna MS, Hemal AK, Dogra PN, Seth A. Impact of imaging and thrombus level in management of renal cell carcinoma extending to veins. Urol Int. 2004;72:129-34.

28. Cuevas C, Raske M, Bush WH, Takayama T, Maki JH, Kolokythas O, Meshberg E. Imaging primary and secondary tumor thrombus of the inferior vena cava: multi-detector computed tomography and magnetic resonance imaging. Curr Probl Diagn Radiol. 2006:35:90-101.

29. Lawrentschuk N, Gani J, Riordan R, Esler S, Bolton DM. Multidetector computed tomography vs magnetic resonance imaging for defining the upper limit of tumour thrombus in renal cell carcinoma: a study and review. BJU Int. 2005;96:291-5.

30. Takeuchi M, Matsuzaki K, Harada M. Carcinosarcoma of the uterus: MRI findings including diffusion-weighted imaging and MR spectroscopy. Acta Radiol. 2016;57:1277-84.
31. Huang YT, Chang CB, Yeh CJ, Lin G, Huang HJ, Wang CC, Lu KY, Ng KK, Yen TC, Lai CH. Diagnostic accuracy of 3.0T diffusion-weighted MRI for patients with uterine carcinosarcoma: assessment of tumor extent and lymphatic metastasis. J Magn Reson Imaging. 2018. https://doi.org/10.1002/jmri.25981.

32. Caivano R, Rabasco P, Lotumolo A, D'Antuono F, Zandolino A, Villonio A, Macarini L, Guglielmi G, Salvatore M, Cammarota A. Gastric cancer: the role of diffusion weighted imaging in the preoperative staging. Cancer Investig. 2014;32:184-90.

33. Delli Pizzi A, Cianci R, Genovesi D, Esposito G, Timpani M, Tavoletta A, Pulsone P, Basilico R, Gabrielli D, Rosa C, et al. Performance of diffusionweighted magnetic resonance imaging at 3.0T for early assessment of tumor response in locally advanced rectal cancer treated with preoperative chemoradiation therapy. Abdom Radiol. 2018. https://doi.org/10.1007/ s00261-018-1457-8.

34. Wang F, Wu LM, Hua XL, Zhao ZZ, Chen XX, Xu JR. Intravoxel incoherent motion diffusion-weighted imaging in assessing bladder cancer invasiveness and cell proliferation. J Magn Reson Imaging. 2018;47:1054-60.

35. Ota T, Hori M, Onishi H, Sakane M, Tsuboyama T, Tatsumi M, Nakamoto A, Kimura T, Narumi Y, Tomiyama N. Preoperative staging of endometrial cancer using reduced field-of-view diffusion-weighted imaging: a preliminary study. Eur Radiol. 2017;27:5225-35.

36. Ogura K, Maekawa S, Okubo K, Aoki Y, Okada T, Oda K, Watanabe Y, Tsukayama C, Arai Y. Dynamic endorectal magnetic resonance imaging for local staging and detection of neurovascular bundle involvement of prostate cancer: correlation with histopathologic results. Urology. 2001;57:721-6.

37. Luzurier A, Jouve De Guibert PH, Allera A, Feldman SF, Conort P, Simon JM, Mozer $P$, Comperat E, Boudghene F, Servois V, et al. Dynamic contrastenhanced imaging in localizing local recurrence of prostate cancer after radiotherapy: limited added value for readers of varying level of experience. J Magn Reson Imaging. 2018. https://doi.org/10.1002/jmri.25991.

38. Mazaheri Y, Akin O, Hricak H. Dynamic contrast-enhanced magnetic resonance imaging of prostate cancer: a review of current methods and applications. World J Radiol. 2017:9:416-25.

Ready to submit your research? Choose BMC and benefit from

- fast, convenient online submission

- thorough peer review by experienced researchers in your field

- rapid publication on acceptance

- support for research data, including large and complex data types

- gold Open Access which fosters wider collaboration and increased citations

- maximum visibility for your research: over $100 \mathrm{M}$ website views per year

At BMC, research is always in progress.

Learn more biomedcentral.com/submissions 